\title{
Association between atopic disease and anemia in pediatrics: a cross-sectional study
}

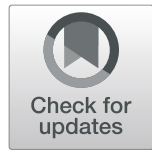

Kiyon Rhew ${ }^{1,2}$ and Jung Mi Oh ${ }^{1,3^{*}}$ (D)

\begin{abstract}
Background: Atopic diseases, such as atopic dermatitis, allergic rhinitis, and asthma, are inflammatory diseases common in pediatric patients. This study investigated whether these inflammatory atopic diseases were associated with anemia in pediatrics.

Methods: A cross-sectional study was conducted using a pediatric dataset from the Health Insurance Review and Assessment Service (HIRA) of South Korea in 2016. Multivariable logistic regression, adjusting for demographic covariates was used for analyse the association between atopic disease and iron deficiency anemia (IDA).

Results: A total of 846,718 pediatric patients were included in the study. Of these, 19,594 (2.31\%) had a diagnosis of IDA. The logistic regression analyses including covariates revealed there were association between atopic disease and IDA. The adjusted OR (aOR) of IDA was 1.42 (95\% Cl, 1.37-1.47) for atopic dermatitis, 1.25 (95\% Cl, 1.21-1.29) for allergic rhinitis, and $1.71(95 \% \mathrm{Cl}, 1.65-1.76)$ for asthma. IDA was more prevalent in patients with multiple comorbid atopic diseases, with aOR of $1.30(95 \% \mathrm{Cl}, 1.25-1.35), 1.81$ (95\% Cl, 1.73-1.89), and 2.58 (95\% Cl, 2.43-2.73) for 1, 2, or 3 atopic diagnoses. There was no evidence of multicollinearity among covariates.

Conclusions: Our findings suggest that atopic disease was associated with IDA. Further study is needed to clarify the distinction between IDA and/or Al to better understand the cause of anemia in patients with inflammatory diseases.
\end{abstract}

Keywords: Atopic disease, Anemia, Allergic rhinitis, Asthma, Atopic dermatitis

\section{Background}

Atopic diseases, such as atopic dermatitis, allergic rhinitis, and asthma, are inflammatory diseases common in pediatrics $[1,2]$. Atopic diseases are often comorbid with other diseases; indeed, the prevalence of infectious disease, autoimmune disease, and mental illness is higher in these patients than those without atopic disease [3-5], and these conditions, singly or in combination, can decrease patients' quality of life and increase morbidity and mortality [6-8].

No study has definitively evaluated whether the persistent inflammatory state of atopic disease impacts the risk of developing anemia. Although one study reported an association between higher iron levels and a lower prevalence of asthma [9], the design was cross-sectional,

\footnotetext{
* Correspondence: jmoh@snu.ac.kr

${ }^{1}$ College of Pharmacy, Seoul National University, 1 Gwanak-ro, Gwanak-gu, Seoul 08826, Republic of Korea

${ }^{3}$ Research Institute of Pharmaceutical Sciences, Seoul National University, 1 Gwanak-ro, Gwanak-gu, Seoul 08826, Republic of Korea

Full list of author information is available at the end of the article
}

making cause and effect difficult to discern. Another study showed that the prevalence of anemia in children with atopic disease is higher than in those without atopic disease [10], but these findings relied on caregiver questionnaires, and the effects of other diseases or drugs that simultaneously affect anemia and atopic disease were not partitioned.

Therefore, in this study, we investigated whether the chronic inflammatory state of atopic patients affects their risk for anemia while controlling for potentially confounding covariates. We considered the risk factors for both atopic disease and anemia.

\section{Methods}

\section{Study population}

South Korea has a universal health insurance system, into which approximately $98 \%$ of all citizens are enrolled. We used a dataset, Health Insurance Review and Assessment Service - Pediatric Patient Sample, (HIRA- 
PPS-2016), that randomly stratified $10 \%$ of the pediatric patients $(\sim 1,100,000)$ under 20 years of age who used a medical institution during the year 2016 [11]. HIRA reported that almost $90 \%$ of Koreans visit medical institutions at least once a year [11]. In South Korea, health care providers report diagnostic codes when prescribing a medication or procedure, and patients are required to physically visit their physician to receive prescriptions. Thus, the requirement of diagnostic codes and absence of refill and telemedicine models results in a dataset with no missing diagnostic codes in the claim data.

The inclusion criterion in this study was patient age under 18 years. Exclusion criteria for this study were: 1) patients who were diagnosed with an anemia other than IDA, 2) patients who were diagnosed with two or more types of anemia. We chose IDA to evaluate if inflammation of atopic diseases increases the prevalence of anemia since the hematologic characteristics of IDA is similar with anemia of inflammation (AI). In addition, other types of anemia except IDA, such as hemolytic anemia, aplastic anemia or folate deficiency anemia, were excluded in this study because of their low prevalence and different mechanisms of etiology. Diagnosis of AI was very low prevalence in children and used for patients with specific chronic condition such as CKD or cancer, so it was also excluded in this study.

\section{Definition of diseases}

We included atopic dermatitis, allergic rhinitis, and asthma as atopic disease based on the presence of the diagnostic code corresponding to one or more of these diseases. Diagnostic codes for these diseases and covariate diseases (see below) are based on The Korean Standard Classification of Disease and Cause of Death-7 (KCD-7) was used for definition of disease (Additional file 1: Table S1). The KCD-7 reflects the update of the International Classification of Diseases 10th Revision (ICD-10) and refines the Korean subtypes of disease and rare diseases.

\section{Covariates}

Covariates were selected based on patient characteristics and common risk factors for anemia and atopic disease. Patient characteristics (age, sex, and insurance type), medical conditions, and medication were included in our analyses as covariates. We included systemic infection, chronic kidney disease (CKD), mental disorder, chronic inflammation, and cancer as covariates for medical condition. Systemic steroids, cyclosporine, and methotrexate were included as covariates for medications. Specific diseases and corresponding KCD-7 codes are listed in Additional file 1: Table S1.

\section{Statistical analysis}

We estimated crude odds ratio (cOR) and adjusted odds ratio (aOR) with 95\% confidence interval (CI). We utilized stepwise multivariate binary logistic regression analysis, including the covariates described above. Multicollinearity test were performed since we included quite a few variables as covariates. All statistical analyses were performed in SAS 9.4 (SAS Institute Inc., Cary, NC, USA), and results were considered statistically significant if the $P$-value was less than 0.05 .

\section{Results}

Out of 1,004,866 patients included in the HIRA-PPS2016, 148,459 patients were older than 18,9551 were diagnosed with anemia other than IDA, and 138 were had more than one type of anemia diagnosis including IDA. Therefore, we included 846,718 pediatric patients (Fig. 1). The prevalence of all anemia was $3.46 \%$ (29,283 patients) in the subjects. The IDA group comprised $2.31 \%(19,594$ patients). The OR of IDA was higher in patients younger $[\mathrm{COR}=0.89(95 \% \mathrm{CI}, 0.89-0.89) ; P<0.001)]$, if age is as continuous variable, in female patients $[\mathrm{COR}=1.20(95 \%$ CI, 1.17-1.24) relative to male patients; $\mathrm{P}<0.001)$ ], and in Medical aid patients [COR $=1.20$ (95\% CI, 1.04-1.23; $P=0.006)$ relative to patients under health insurance program. All covariates, including systemic infection, mental disorder, CKD, chronic inflammation, cancer, and medication (steroid, methotrexate, and cyclosporine) indicated significantly higher ORs of IDA (Table 1).

\section{Association of atopic disease and anemia}

We found that the aOR of IDA was significantly higher across patients with atopic disease, at $1.42(95 \% \mathrm{CI}$, $1.37-1.47 ; \quad P<0.001), 1.25$ (95\% CI, 1.21-1.29; $\mathrm{P}<$ $0.001)$, and $1.71(95 \% \mathrm{CI}, 1.65-1.76 ; \mathrm{P}<0.001)$ in patients with atopic dermatitis, allergic rhinitis, and asthma, respectively. All three atopic diseases demonstrated a significant, positive association with IDA before and after applying covariates (Table 2).

Multiple atopic diseases in a single patient had higher associations with IDA than a single diagnosis. The aOR for 1 atopic disease and IDA was 1.30 (95\% CI, 1.25$1.35 ; P<0.001)$, increased to 1.81 (95\% CI, 1.73-1.89); $P<.001)$ in those with 2 atopic diseases, and was highest in patients with 3 atopic diseases $[\mathrm{aOR}=2.58(95 \% \mathrm{CI}$, 2.43-2.73; $\mathrm{P}<0.001$ )] (Table 2). We also reported full multivariable logistic regression models of association between IDA and atopic disease in Additional file 1: Table S2. In addition, there was no evidence of multicollinearity among covariates. Maximum VIF (variance inflation factor) was lower than 2.0, and lowest eigenvalue was 0.32 . 


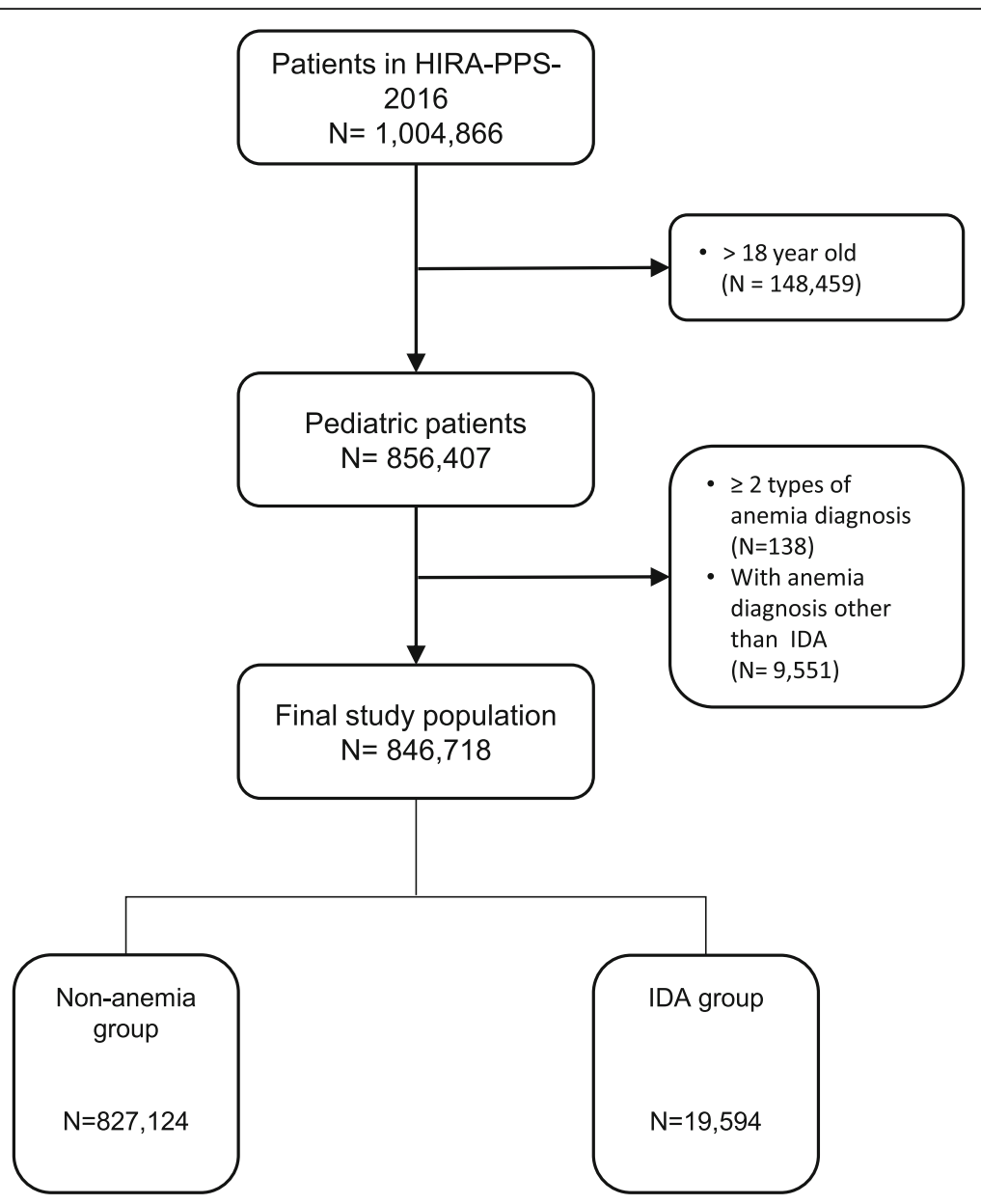

Fig. 1 Flow diagram for study subject inclusion

\section{Discussion}

In this study, we showed that prevalence of IDA was associated with three atopic diseases (atopic dermatitis, allergic rhinitis, and asthma) by a large-scale data analysis of nearly 850,000 South Korean pediatric patients. We also found that the aOR of IDA was increased in children with multiple atopic disease diagnoses. Our results showed that comorbid medical conditions, the use of medication, gender, age, and socio-economic level were associated with the prevalence of IDA.

The prevalence of anemia in pediatric patients was approximately $3.42 \%$ in this study, the majority of which was IDA (2.31\%); this figure is lower than the results of existing epidemiology studies, which suggest rates between 4 and $6 \%[12,13]$. The apparently low diagnosis of anemia in our study may be due to the use of the HIRA-PPS-2016 dataset because, in South Korea, most health screenings for infants and young children are brief physical examinations and do not routinely involve testing for anemia.

The etiology of anemia varies. AI is an anemia caused by inflammatory immune activation $[14,15]$. AI produces similar hematological findings to iron deficiency anemia (IDA), including low hemoglobin and iron levels, and shows similar clinical manifestation to anemia in patients with chronic obstructive pulmonary disease, CKD, and congestive heart failure $[16,17]$. We hypothesized that inflammatory conditions in patients with atopic diseases would increase their risk of anemia because previous studies have shown that inflammation, CKD, cancer, and systemic infection correlate with AI [18-22]. Indeed, in our study, the association of these diseases and medication was also high in patients diagnosed with IDA.

Inflammatory disease, malignancy, and infections are the major medical conditions underlying AI. In addition, combined anemia of IDA and AI is typically presented in patients with irritable bowel disease (IBD), or gastrointestinal (GI) malignancy [23]. Inflammatory diseases or depression would be a confounding factor which impact on both risk of anemia and atopic disease. Patients with moderate to severe atopic disease were treated by immunosuppressant such as methotrexate, cyclosporine, or steroids. Those medications could cause bleeding, anemia, or hematologic 
Table 1 Patient characteristics according to iron deficiency anemia

\begin{tabular}{|c|c|c|c|c|}
\hline & \multicolumn{2}{|l|}{ Frequency (\%) } & \multirow[t]{2}{*}{ Crude OR (95\% Cl) } & \multirow[t]{2}{*}{$P$ value } \\
\hline & No anemia $N=(827,124)$ & $\begin{array}{l}\text { IDA } \\
(N=19,594)\end{array}$ & & \\
\hline Age & $8.97(5.16)^{a}$ & $5.98(5.48)^{a}$ & $0.89(0.89-0.89)$ & $<.001$ \\
\hline \multicolumn{5}{|l|}{ Sex } \\
\hline Male & $428,361(51.79)$ & $9253(47.22)$ & 1 [Reference] & \\
\hline Female & $398,763(48.21)$ & $10,341(52.78)$ & $1.20(1.17-1.24)$ & $<.001$ \\
\hline \multicolumn{5}{|l|}{ Insurance Types } \\
\hline Health insurance & $806,892(97.55)$ & 19,054 (97.24) & 1 [Reference] & \\
\hline Medical aid & $20,232(2.45)$ & $540(2.76)$ & $1.13(1.04-1.23)$ & 0.006 \\
\hline \multicolumn{5}{|l|}{ Systemic infection } \\
\hline Meningitis & $1645(0.20)$ & $130(0.66)$ & $3.35(2.80-4.01)$ & $<.001$ \\
\hline BJ & $803(0.10)$ & $41(0.21)$ & $2.16(1.58-2.96)$ & $<.001$ \\
\hline Sepsis & $3071(0.37)$ & $478(2.44)$ & $6.71(6.09-7.40)$ & $<.001$ \\
\hline HEP & $2445(0.30)$ & $695(3.55)$ & $12.40(11.39-13.51)$ & $<.001$ \\
\hline CKD & $57(0.01)$ & $26(0.13)$ & $19.28(12.12-30.67)$ & $<.001$ \\
\hline \multicolumn{5}{|l|}{ Mental disorder } \\
\hline Depression & $3931(0.48)$ & $178(0.91)$ & $1.92(1.65-2.23)$ & $<.001$ \\
\hline Anxiety & $6518(0.79)$ & $309(1.58)$ & $2.02(1.80-2.26)$ & $<.001$ \\
\hline \multicolumn{5}{|l|}{ Chronic inflammation } \\
\hline PUD & $15,248(1.84)$ & $640(3.27)$ & $1.80(1.66-1.95)$ & $<.001$ \\
\hline COPD & $12,650(1.53)$ & $422(2.15)$ & $1.42(1.29-1.56)$ & $<.001$ \\
\hline SLE & $228(0.03)$ & $26(0.13)$ & $4.83(3.22-7.25)$ & $<.001$ \\
\hline RA & $1454(0.18)$ & $96(0.49)$ & $2.80(2.27-3.44)$ & $<.001$ \\
\hline $\mathrm{IBD}$ & $265(0.03)$ & $68(0.35)$ & $10.87(8.32-14.19)$ & $<.001$ \\
\hline Cancer & $1147(0.14)$ & $133(0.68)$ & $4.92(4.11-5.89)$ & $<.001$ \\
\hline \multicolumn{5}{|l|}{ Medication } \\
\hline Steroid & $333,265(40.29)$ & $11,726(59.84)$ & $2.21(2.15-2.27)$ & $<.001$ \\
\hline Methotrexate & $162(0.02)$ & $26(0.13)$ & $6.78(4.48-10.27)$ & $<.001$ \\
\hline Cyclosporine & $302(0.04)$ & $29(0.15)$ & $4.07(2.78-5.96)$ & $<.001$ \\
\hline
\end{tabular}

${ }^{\mathrm{a}}$ mean (standard deviation)

IDA Iron deficiency anemia, $B$ J Bone and joint infection, HEP Hepatitis, CKD Chronic kidney disease, PUD Peptic ulcer disease, COPD Chronic obstructive pulmonary disease, SLE Systemic lupus erythematosus, RA Rheumatoid arthritis, IBD Irritable bowel disease

disorders [24-26]. Therefore, we included those medical condition and medication as covariates to adjust for minimizing their effects on outcome.

Most covariates we included were still associated with increased prevalence of IDA. Chronic obstructive pulmonary disease (COPD) and methotrexate, however, showed null effects after adjusting all covariates. As is well known, COPD is a disease that increases in incidence after middle age. We only included pediatric patients in this study, so it may have led to differences in outcomes from other inflammatory diseases in the outcome of COPD. Methotrexate is an antimetabolite and antifolate, which leads low level of folic acid in the body. Hematologic toxicity such as anemia in the methotrexate users would be more like folate deficiency anemia not IDA.
Because AI has a hematological manifestation similar to IDA [27], hepcidin is a useful indicator to differentiate AI from IDA $[28,29]$. Unfortunately, hepcidin is rarely assessed in common clinical settings. Therefore, patients with AI may be diagnosed with IDA instead of AI, which would result in an artificially inflated risk of IDA in atopic disease patients.

In this study, we found that multiple atopic disease diagnoses in a patient had stronger associations with IDA, which is consistent with previous research [14]. Notably, Drury's study showed allergic rhinitis was not associated with anemia. Our study indicated that the aOR of allergic rhinitis, although statistically significant, was lower than that for other atopic diseases (asthma and atopic dermatitis). The effect of systemic inflammation may therefore differ among atopic diseases. 
Table 2 Association between atopic diseases and iron deficiency anemia

\begin{tabular}{|c|c|c|c|c|c|c|}
\hline \multirow[t]{2}{*}{ Atopic diseases } & \multicolumn{2}{|l|}{ Frequency (\%) } & \multirow{2}{*}{$\begin{array}{l}\text { Crude OR (95\% } \\
\text { Cl) }\end{array}$} & \multirow[t]{2}{*}{$P$ value } & \multirow{2}{*}{$\begin{array}{l}\text { Adjusted OR } \\
(95 \% \mathrm{Cl})\end{array}$} & \multirow[t]{2}{*}{$P$ value } \\
\hline & $\begin{array}{l}\text { No anemia } \\
N=(827,124)\end{array}$ & $\begin{array}{l}\text { IDA } \\
(N=19,594)\end{array}$ & & & & \\
\hline \multicolumn{7}{|l|}{ Atopic dermatitis } \\
\hline No & $722,427(87.34)$ & $14,853(75.80)$ & 1 [Reference] & & 1 [Reference] & \\
\hline Yes & $104,697(12.66)$ & $4741(24.20)$ & $2.20(2.13-2.28)$ & $<.001$ & $1.42(1.37-1.47)$ & $<.001$ \\
\hline \multicolumn{7}{|l|}{ Allergic rhinitis } \\
\hline No & $534,731(64.65)$ & $10,344(52.79)$ & 1 [Reference] & & 1 [Reference] & \\
\hline Yes & 292,393 (35.35) & $9250(47.21)$ & $1.64(1.59-1.68)$ & $<.001$ & $1.25(1.21-1.29)$ & $<.001$ \\
\hline \multicolumn{7}{|l|}{ Asthma } \\
\hline No & $600,218(72.57)$ & $9393(47.94)$ & 1 [Reference] & & 1 [Reference] & \\
\hline Yes & $226,906(27.43)$ & $10,201(52.06)$ & $2.87(2.79-2.96)$ & $<.001$ & $1.71(1.65-1.76)$ & $<.001$ \\
\hline \multicolumn{7}{|c|}{ Atopic diseases, No. } \\
\hline 0 & $388,310(46.95)$ & $5170(26.39)$ & 1 [Reference] & & 1 [Reference] & \\
\hline 1 & $280,108(33.87)$ & 6638 (33.88) & $1.78(1.72-1.85)$ & $<.001$ & $1.30(1.25-1.35)$ & $<.001$ \\
\hline 2 & $132,230(15.99)$ & $5804(29.62)$ & $3.30(3.17-3.42)$ & $<.001$ & $1.81(1.73-1.89)$ & $<.001$ \\
\hline 3 & $26,476(3.20)$ & $1982(10.12)$ & $5.62(5.33-5.93)$ & $<.001$ & $2.58(2.43-2.73)$ & $<.001$ \\
\hline
\end{tabular}

IDA Iron deficiency anemia

Adjusted by age, sex, insurance type, meningitis, bone and joint infection, sepsis, hepatitis, depression, anxiety, chronic kidney disease, peptic ulcer disease,

chronic obstructive pulmonary disease, systemic lupus erythematosus, rheumatoid arthritis, irritable bowel disease, cancer, steroid, methotrexate, cyclosporine

We confirmed that the prevalence of IDA in pediatric patients with atopic dermatitis, allergic rhinitis, and/or asthma is higher than in patients without atopic disease. Because previous research has not provided evidence that atopic patients are at risk for anemia, monitoring and treatment guidelines for anemia in pediatric patients with atopic disease do not exist. However, treatment for anemia in children is crucial because of its potential to reduce quality of life [30], increase morbidity [31-34], and negatively affect school performance and physical growth $[35,36]$. Therefore, we suggest that appropriate treatment of patients with atopic disease may require assessment for and treatment of IDA or AI.

Our study has several limitations. First, it is a crosssectional study, and, thus, we cannot determine a causative relationship between atopic disease and anemia. However, the reverse causation-that anemia causes atopic disease-is unlikely given the pathophysiology of anemia and atopic disease. Second, because of its similarities to IDA, we have not directly assessed AI, nor have we identified whether the inflammatory state of atopic disease causes AI. Third, we cannot completely rule out selection bias because healthy children are more likely not to have screening for anemia. However, HIRA reported that most of the population use medical service at least once a year. In addition, unlike other serious health condition or medication use we included as covariates, anemia testing is not common in children with atopic disease, so the impact on outcomes would be limited. Nevertheless, this study has strengths, such as the inclusion of medical conditions and medications as covariates. We used diagnostic code, not self-report, for definition of disease. Lastly, by utilizing a large dataset sampled from national healthcare claims, we were able to objectively assess the association between atopic disease and anemia.

\section{Conclusions}

We suggest that the inflammatory conditions that characterize atopic diseases (atopic dermatitis, allergic rhinitis, and asthma) could associated with IDA and/or AI. Further study is needed to clarify the distinction between IDA and AI to better understand the cause of anemia in patients with inflammatory diseases.

\section{Supplementary information}

Supplementary information accompanies this paper at https://doi.org/10. 1186/s12887-019-1836-5.

Additional file 1: Table S1. The list of KCD-7 diagnostic codes of diseases in the study. Table S2. Adjusted odds ratio of IDA for all covariates in final model.

\section{Abbreviations}

Al: Anemia of inflammation; aOR: Adjusted odds ratio; Cl: Confidence intervals; CKD: Chronic kidney disease; COPD: Chronic obstructive pulmonary disease; cOR: Crude odds ratio; Gl: Gastrointestinal; HIRA-PPS: Health Insurance Review and Assessment Service-Pediatric Patient Sample; IBD: Irritable bowel disease; ICD-10: International Classification of Diseases 10th Revision; IDA: Iron deficiency anemia; KCD-7: The Korean Standard Classification of Disease and Cause of Death-7; OR: Odds ratio 


\section{Acknowledgements}

This study was conducted by the support of the research year system of Dongduk Women's University in 2018.

\section{Authors' contributions}

KR defined the research question, and designed the method. KR analyzed the data and write the first manuscript. JO interpreted the results and review and edit the paper. Both authors approved the manuscript.

\section{Funding}

None.

\section{Availability of data and materials}

The datasets analysed during the current study are available from the corresponding author on reasonable request.

\section{Ethics approval and consent to participate}

The study was approved by the institutional review board at Dongduk Women's University.

\section{Consent for publication}

Not applicable.

\section{Competing interests}

The authors declare that they have no competing interests.

\section{Author details}

${ }^{1}$ College of Pharmacy, Seoul National University, 1 Gwanak-ro, Gwanak-gu, Seoul 08826, Republic of Korea. ${ }^{2}$ College of Pharmacy, Dongduk Women's University, 60 Hwarang-ro 13-gil, Seongbuk-gu, Seoul 02748, Republic of Korea. ${ }^{3}$ Research Institute of Pharmaceutical Sciences, Seoul National University, 1 Gwanak-ro, Gwanak-gu, Seoul 08826, Republic of Korea.

Received: 12 July 2019 Accepted: 15 November 2019

Published online: 25 November 2019

\section{References}

1. Hill DA, Grundmeier RW, Ram G, Spergel JM. The epidemiologic characteristics of healthcare provider-diagnosed eczema, asthma, allergic rhinitis, and food allergy in children: a retrospective cohort study. BMC Pediatr. 2016:16:133.

2. Hameed R, Peters RL, Field MJ, Koplin JJ, Dharmage SC, Allen KJ. Selfreported asthma prevalence and control in a population-based cohort of Australian school students aged 10-14 years. Arch Dis Child. 2019;104:612-3.

3. Pols DHJ, Bohnen AM, Nielen MMJ, Korevaar JC, Bindels PJE. Risks for comorbidity in children with atopic disorders: an observational study in Dutch general practices. BMJ Open. 2017;7:e018091.

4. Becker-Haimes EM, Diaz KI, Haimes BA, Ehrenreich-May J. Anxiety and atopic disease: comorbidity in a youth mental health setting. Child Psychiatry Hum Dev. 2017:48:528-36.

5. Narla S, Silverberg Jl. Association between atopic dermatitis and autoimmune disorders in US adults and children: a cross-sectional study. J Am Acad Dermatol. 2019:80:382-9.

6. Karimkhani C, Dellavalle RP, Coffeng LE, et al. Global skin disease morbidity and mortality: an update from the global burden of disease study 2013. JAMA Dermatol. 2017;153:406-12.

7. Ebmeier S, Thayabaran D, Braithwaite I, Bénamara C, Weatherall M, Beasley R. Trends in international asthma mortality: analysis of data from the WHO mortality database from 46 countries (1993-2012). Lancet. 2017:390:935-45.

8. Ponte EV, Cruz AA, Athanazio R, et al. Urbanization is associated with increased asthma morbidity and mortality in Brazil. Clin Respir J. 2018;12:410-7.

9. Brigham EP, McCormack MC, Takemoto CM, Matsui EC. Iron status is associated with asthma and lung function in US women. PLoS One. 2015;10:e0117545.

10. Drury KE, Schaeffer M, Silverberg Jl. Association between atopic disease and anemia in US children. JAMA Pediatr. 2016;170:29-34.

11. Kim L, Kim JA, Kim S. A guide for the utilization of Health Insurance Review and Assessment Service national patient samples. Epidemiol Health. 2014; 36:e2014008.

12. Tezera R, Sahile Z, Yilma D, Misganaw E, Mulu E. Prevalence of anemia among school-age children in Ethiopia: a systematic review and metaanalysis. Syst Rev. 2018;7:80
13. Stevens GA, Finucane MM, De-Regil LM, et al. Global, regional, and national trends in haemoglobin concentration and prevalence of total and severe anemia in children and pregnant and non-pregnant women for 1995-2011: a systematic analysis of population-representative data. Lancet Glob Health. 2013;1:e16-25

14. Metzgeroth $\mathrm{G}$, Hastka J. Iron deficiency anemia and anemia of chronic disorders. Internist (Berl). 2015;56:978-88.

15. Weiss G. Iron metabolism in the anemia of chronic disease. Biochim Biophys Acta. 2009:1790:682-93.

16. Silverberg DS. The role of erythropoiesis stimulating agents and intravenous (IV) iron in the cardio renal anemia syndrome. Heart Fail Rev. 2011:16:609-14.

17. Ferrucci L, Semba RD, Guralnik JM, et al. Proinflammatory state, hepcidin, and anemia in older persons. Blood. 2010;115:3810-6.

18. van Eijk LT, Kroot JJ, Tromp M, van der Hoeven JG, Swinkels DW, Pickkers P. Inflammation-induced hepcidin-25 is associated with the development of anemia in septic patients: an observational study. Crit Care. 2011;15:R9.

19. Murawska N, Fabisiak A, Fichna J. Anemia of chronic disease and iron deficiency anemia in inflammatory bowel diseases: pathophysiology, diagnosis, and treatment. Inflamm Bowel Dis. 2016;22:1198-208.

20. Guidi GC, Lechi SC. Advancements in anemias related to chronic conditions. Clin Chem Lab Med. 2010;48:1217-26.

21. Cappellini MD, Comin-Colet J, de Francisco A, et al. Iron deficiency across chronic inflammatory conditions: international expert opinion on definition, diagnosis, and management. Am J Hematol. 2017;92:1068-78.

22. Macciò A, Madeddu C, Gramignano G, et al. The role of inflammation, iron, and nutritional status in cancer-related anemia: results of a large, prospective, observational study. Haematologica. 2015;100:124-32.

23. Nairz $\mathrm{M}$, Theurl I, Wolf $\mathrm{D}$, et al. Iron deficiency or anemia of inflammation? : Differential diagnosis and mechanisms of anemia of inflammation. Wien Med Wochenschr. 2016;166:411-23.

24. Narum S, Westergren T, Klemp M. Corticosteroids and risk of gastrointestinal bleeding: a systematic review and meta-analysis. BMJ Open. 2014;4(5):e004587.

25. Dubey L, Chatterjee S, Ghosh A. Hepatic and hematological adverse effects of long-term low-dose methotrexate therapy in rheumatoid arthritis: an observational study. Indian J Pharm. 2016:48:591-4.

26. Garratty G. Immune hemolytic anemia associated with drug therapy. Blood Rev. 2010:24:143-50

27. Polin $V$, Coriat $R$, Perkins $G$, et al. Iron deficiency: from diagnosis to treatment. Dig Liver Dis. 2013:45:803-9.

28. Theurl I, Aigner E, Theurl M, et al. Regulation of iron homeostasis in anemia of chronic disease and iron deficiency anemia: diagnostic and therapeutic implications. Blood. 2009;113:5277-86.

29. Wang CY, Babitt JL. Hepcidin regulation in the anemia of inflammation. Curr Opin Hematol. 2016:23:189-97.

30. Strauss WE, Auerbach M. Health-related quality of life in patients with iron deficiency anemia: impact of treatment with intravenous iron. Patient Relat Outcome Meas. 2018;9:285-98.

31. Bansal K, Goyal M, Dhingra R. Association of severe early childhood caries with iron deficiency anemia. J Indian Soc Pedod Prev Dent. 2016;34:36-42.

32. Schieffer KM, Connor JR, Pawelczyk JA, Sekhar DL. The relationship between iron deficiency anemia and sensorineural hearing loss in the pediatric and adolescent population. Am J Audiol. 2017;26:155-62

33. Chen MH, Su TP, Chen YS, et al. Association between psychiatric disorders and iron deficiency anemia among children and adolescents: a nationwide population-based study. BMC Psychiatry. 2013;13:161.

34. Hartfield DS, Tan J, Yager JY, et al. The association between iron deficiency and febrile seizures in childhood. Clin Pediatr (Phila). 2009:48:420-6.

35. Pivina L, Semenova Y, Doşa MD, Dauletyarova M, Bjørklund G. Iron deficiency, cognitive functions, and neurobehavioral disorders in children. J Mol Neurosci. 2019;68:1-10.

36. Allali S, Brousse V, Sacri AS, Chalumeau M, de Montalembert M. Anemia in children: prevalence, causes, diagnostic work-up, and long-term consequences. Expert Rev Hematol. 2017:10:1023-8.

\section{Publisher's Note}

Springer Nature remains neutral with regard to jurisdictional claims in published maps and institutional affiliations. 